\title{
Coupling MD Simulations and X-ray Absorption Spectroscopy to Study lons in Solution
}

Cite as: AIP Conference Proceedings 963, 621 (2007); https://doi.org/10.1063/1.2827049

Published Online: 03 December 2007

E. Sánchez Marcos, E. C. Beret, J. M. Martínez, R. R. Pappalardo, R. Ayala, and A. Muñoz-Páez

Export citation

\section{Lock-in Amplifiers up to $600 \mathrm{MHz}$

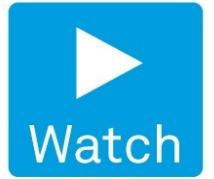

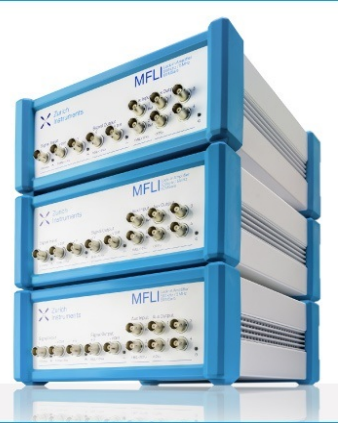




\title{
Coupling MD Simulations and X-ray Absorption Spectroscopy to Study Ions in Solution
}

\author{
E. Sánchez Marcos*, E.C. Beret*, J.M. Martínez *, R.R. Pappalardo *, R. Ayala ${ }^{\dagger}$ and \\ A. Muñoz-Páez. ${ }^{\dagger}$ \\ ${ }^{*}$ University of Seville. Dept. of Physical Chemistry. \\ $\dagger$ University of Seville. CSIC-ICMSE. Dept. of Inorganic Chemistry.
}

\begin{abstract}
The structure of ionic solutions is a key-point in understanding physicochemical properties of electrolyte solutions. Among the reduced number of experimental techniques which can supply direct information on the ion environment, $\mathrm{X}-$ ray Absorption techniques (XAS) have gained importance during the last decades although they are not free of difficulties associated to the data analysis leading to provide reliable structures. Computer simulations of ions in solution is a theoretical alternative to provide information on the solvation structure. Thus, the use of computational chemistry can increase the understanding of these systems although an accurate description of ionic solvation phenomena represents nowadays a significant challenge to theoretical chemistry. We present: (a) the assignment of features in the XANES spectrum to well defined structural motif in the ion environment, (b) MD-based evaluation of EXAFS parameters used in the fitting procedure to make easier the structural resolution, and (c) the use of the agreement between experimental and simulated XANES spectra to help in the choice of a given intermolecular potential for Computer Simulations. Chemical problems examined are: (a) the identification of the second hydration shell in dilute aqueous solutions of highly-charged cations, such as $\mathrm{Cr}^{3+}, \mathrm{Rh}^{3+}$, $\mathrm{Ir}^{3+}$, (b) the invisibility by XAS of certain structures characterized by Computer Simulations but exhibiting high dynamical behavior and (c) the solvation of $\mathrm{Br}^{-}$in acetonitrile.
\end{abstract}

Keywords: Aqueous solution; Ionic hydration; Molecular Dynamics, EXAFS, XANES, spectra simulation.

PACS: $61.20 . \mathrm{Gy}, 61.20 . \mathrm{Qg}, 61.20 . \mathrm{Ja}, 78.70 \mathrm{Dm}$

\section{INTRODUCTION.}

The main experimental techniques for structural studies of ionic solutions are X-ray diffraction (XRD), neutron diffraction (ND) and X-ray absorption spectroscopy (XAS). Although XRD and ND can yield structural information about disordered systems like most liquids, their accuracy decreases strongly in the case of multicomponent systems (XRD) or requires considerable synthetic efforts to perform the isotopic substitutions (ND). These facts along with the need of relatively high concentrations preclude to certain extent their use. Because XAS is element-specific, the local structure around one type of atom can be solved, additionally, it can be applied to a wide range of concentrations, ranging typically from a few millimolar to several molar. Then, XAS represents the most suitable technique to supply direct structural information on highly dilute electrolyte solutions.

The X-ray Absorption spectrum has been traditionally divided into two energy regions. The low energy part of the spectrum, extending up to $\sim 50-100 \mathrm{eV}$ above the absorbing edge, called X-ray Absorption Near Edge Structure (XANES) region and the high energy part of the spectrum (over $100 \mathrm{eV}$ above the threshold up to approximately 1000 eV) called Extended X-ray Absorption Fine Structure (EXAFS). Since the 1980s, one of the XAS techniques, extended $\mathrm{X}$-ray absorption fine structure (EXAFS), has been applied to determine the pseudo partial pair correlation functions of atoms in both crystalline and amorphous systems. The main limitation of the technique is the high correlation among factors affecting the amplitude and the large number of multiple scattering paths which may contribute to the amplitude. The XANES region has been shown to be dependent on many parameters of the system under study: the oxidation state of the absorber atom, bond angles, coordination geometry, distances of the absorbing atom to the first, and higher coordination shells, etc. Conversely to the EXAFS region, there is no simple formulation that encompasses all these parameters. This fact has usually led to the use of XANES information on a more qualitative level that envisages the spectrum as a "fingerprint" of the system under study. In the last years, implementation of models for the absorption phenomena have allowed the development of codes which accurately simulate the XANES region thus approaching the situation for the EXAFS region. This way the theoretical simulation of XANES of ionic solutions is an emerging branch.

CP963, Vol. 1, Computational Methods in Science and Engineering,

Theory and Computation: Old Problems and New Challenges, edited by G. Maroulis and T. Simos

(C) 2007 American Institute of Physics 978-0-7354-0477-9/07/\$23.00 
A strategy to reduce the degree of uncertainty in the analysis of the XAS spectra has been the inclusion of independent information derived from computer simulations for both EXAFS and XANES spectra[1, 2, 3, 4]. Once reliable structural and dynamic information is obtained from computer simulations, the microscopic description of the close environment of the absorber atom allows the independent computation of some of the parameters involved in the spectroscopic data analysis and the assignment of certain features of a given spectrum to particular structural arrangements around it.

The aim of this work is to present a series of structural results derived from experimental and theoretical methods for a series of single ions. Particular attention is devoted to the experimental determination of the second hydration shell of $\mathrm{Cr}(\mathrm{III}), \mathrm{Rh}(\mathrm{III}), \operatorname{Ir}(\mathrm{III})$ and $\mathrm{Pd}(\mathrm{II})$ aquaions. The case of the square-planar Pd(II) aquaion introduces the peculiarity of the possible determination of water molecules in the axial region, called meso-shell [5,6]. Finally, the synergy achieved by combining both spectroscopic and theoretical results improves the analysis process in two ways: (i) by refining the XAS data analysis and (ii) by supporting the intermolecular potential developed as well as the microscopic vision of the hydration derived from computer simulations. These two points will be illustrated by the study of the solvation structure of $\mathrm{Br}^{-}$in acetonitrile, taking the reproduction of the experimental $\mathrm{Br} \mathrm{K}$-edge XANES of this system[7] as the reference to elucidate the most appropriate bromide-acetonitrile intermolecular potential.

\section{METHODOLOGY}

Classical Molecular Dynamics (MD) simulations corresponding to transition metal ions presented in this work are based on the use of the Hydrated Ion (HI) concept to model the cation-water interactions[8]. The high perturbation exerted by the metal ion on the water molecules directly interacting with it, allows the consideration of them from the rest of the solvent. Then, the aquaion $\left[\mathrm{M}\left(\mathrm{H}_{2} \mathrm{O}\right)_{n}\right]^{m+}$ is adopted as the representative entity in solution and the description of the interactions involving the cation is performed through the definition of two interaction potentials: one for the hydrated ion-water (HIW) forces and one for the internal dynamics of the aquaion, i.e. ion-first shell water (IW1) forces[9]. Details for the development of intermolecular potentials of the different cations employed in this work can be found elsewhere (Cr(III),[10, 9] $\mathrm{Rh}(\mathrm{III}),[11] \operatorname{Ir}(\mathrm{III})[4]$ and $\mathrm{Pd}(\mathrm{II})[5])$. In the case of $\mathrm{Pd}(\mathrm{II})$ in water, Car-Parrinello ab initio MD simulations were also carried out for a system formed by one Pd(II) cation and 70 water molecules[12].

The $\mathrm{Br}$-acetonitrile (ACN) interaction potentials tested to describe the solvation of bromide in acetonitrile are built on the basis of the acetonitrile model proposed by Grabuleda et al.[13] combined with the bromide parametrization performed by McCammon et al.[14] (hereafter called Pot. A). A second set of potentials is a modification of the previous one, based on the hydrated ion concept. The quantum-mechanical optimized structure of the $\left[\mathrm{Br}(\mathrm{ACN})_{9}\right]^{-}$ cluster was used as a reference to modify the van der Waals parameter $\sigma_{\mathrm{Br}-\mathrm{H}}$ in such a way that the potentials leads to a similar optimized structure than the quantum-mechanical, (Pot. B). The third set of potentials consist in the modification of the $\sigma_{\mathrm{Br}-\mathrm{H}}$ parameter which was reduced from $3.6 \AA$ for Pot. A, to $3.2 \AA$ for Pot. B and to a value of $2.9 \AA$ (Pot. C). This range allows an insight into the relationships between XANES spectrum and structure. Details of these intermolecular potentials and simulation conditions can be found elsewhere[15].

MD simulations provided snapshosts evenly spaced in time for the analysis of the EXAFS and XANES part. The FEFF 8.10 code developed by Rehr and col.[16] was used to calculate the corresponding individual spectrum generated for each snapshot. The simulated spectrum is obtained by averaging over 1000 individual EXAFS spectrum, whereas for XANES 100 individual spectra are enough.

\section{RESULTS AND DISCUSSION.}

\section{Second hydration shell determination}

The high charge of trivalent transition metal cations, such as $\mathrm{Cr}(\mathrm{III}), \mathrm{Rh}(\mathrm{III})$ or $\operatorname{Ir}(\mathrm{III})$ in aqueous solutions joined with the high kinetic stability of their corresponding aquaions lead to expect a structural order beyond the first hydration shell for these cations. Second hydration shell has been determined for several trivalent and divalent metal cations from X-ray and neutron diffraction techniques for highly concentrated aqueous solutions. More recently, we have proposed that refined analysis of EXAFS data may also give information on the second hydration shell for highly diluted aqueous solutions of trivalent metal cation such as $\mathrm{Cr}(\mathrm{III})$ or $\mathrm{Rh}(\mathrm{III})[17,18,2]$. A paradigm of inert aquaion 

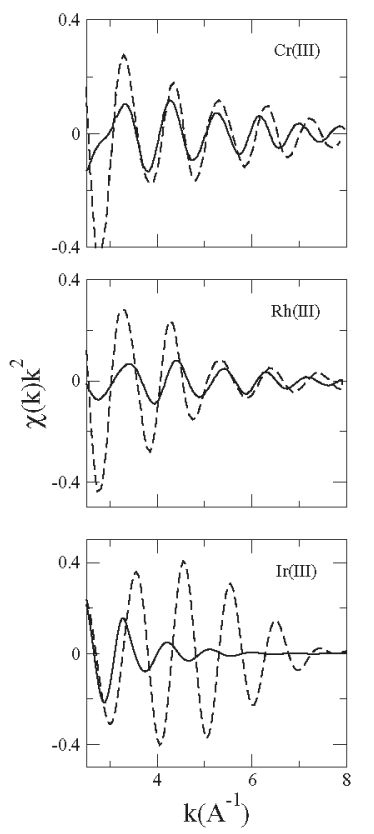

FIGURE 1. Contribution to the $k$-space EXAFS spectrum of the Single Scattering due to the second shell (solid line) and the multiple scattering (dashed line) of the first shell for $\mathrm{Cr}$ (III), Rh(III) and Ir(III) in aqueous solution.

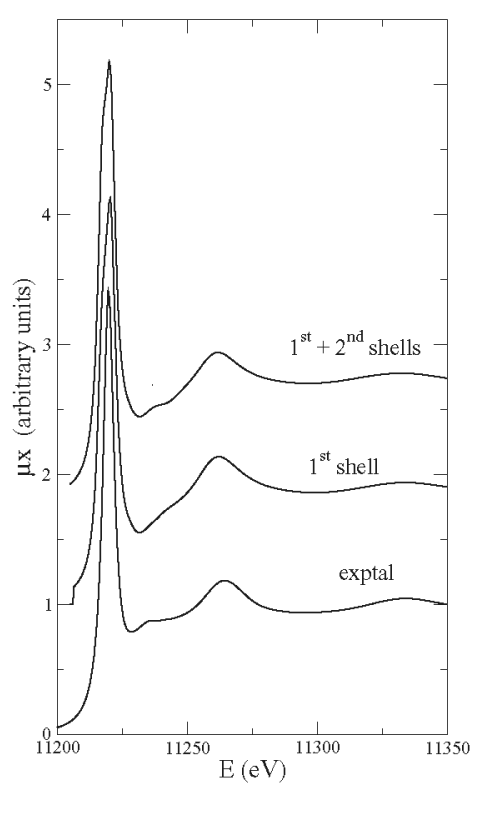

FIGURE 2. Ir L LII-edge XANES experimental and simulated spectra. The simulated ones use a set of snapshots including only the 1 st. shell $\left(R_{\text {cutoff }}=3.1 \AA\right)$ or two hydration shells ( $R_{\text {cutoff }}=6.0 \AA$ ).

is the hexahydrate of Ir(III) that exhibits the longest mean lifetime for the first-shell water molecules at $298 \mathrm{~K}, \sim 300$ years. Contrary to what is expected, the second hydration shell can not be determined from Ir LIII EXAFS spectra. Data analysis of EXAFS function show that the amplitude of the second shell (SS) contribution is too small compared to the first-shell multiple scattering (MS) contribution which appears at similar distances. Figure 1 shows the comparison of these two contributions for the cases of $\mathrm{Cr}$ (III), Rh(III) and $\operatorname{Ir}(\mathrm{III})$. In the two first cases the SS:MS contribution ratio is roughly $1: 2$ whereas for the $\operatorname{Ir}(\mathrm{III})$ this ratio decreases to $1: 4$, thus avoiding the structural determination of the second shell[4]. This differential behavior is due to the heavier nature of the iridium atom compared to rhodium or chromium which results in the backscattering amplitude of the paths including Ir as an intermediate step (i.e. just the MS paths) to become higher than the ones not including it.

Figure 2 shows the experimental Ir $\mathrm{L}_{\mathrm{III}}$ XANES spectrum corresponding to the same dilute $\operatorname{Ir}(\mathrm{III})$ aqueous solutions together with the simulated spectra based on a MD simulation of $\operatorname{Ir}(\mathrm{III})$, two different cutoff radii, $3.1 \AA$ and $6.0 \AA$ for the structure considered for each snapshot, were employed. In the first case, the simulated XANES spectrum is only due to contributions from the first shell, whereas in the second spectrum the second shell contribution is taken into account. Contrary to the EXAFS analysis, the experimental hump appearing $16 \mathrm{eV}$ above the main resonance is well reproduced when the second hydration shell is included in the structures employed to simulate the XANES spectrum from the statistical trajectories. Similar behavior is observed when the XANES spectra of $\mathrm{Cr}$ (III) or Rh(III) aqueous solutions are examined[2]. The spectroscopical reason which hampered the determination of the second hydration in EXAFS for the iridium case is strongly reduced in the case of XANES due to the fact the dependence of the DebyeWaller factor with the vector $k$ has exponential character. Thus, since XANES involves low $k$ values, the sensitivity of this part of the XAS spectrum is much smaller, and features of the second hydration shell become relevant. 


\section{The Invisibility of some hydration regions to XAS}

$\operatorname{Pd}(\mathrm{II})$ cation forms a square planar tetrahydrate in water solution. Due to this fact the second hydration shell adopts a crown-like shape enclosing the aquaion and leaving the axial region (above and below the molecular plane) sparsely populated. Nevertheless, results from Classical MD[5], QM/MM MD[6] and CPMD[12] simulations show the existence of a certain hydration structure in this axial region (meso-shell), its behaviour being far more dynamic than for the second solvation shell. Could such a meso-shell structure be determined by means of XAS techniques? Based on our previous knowledge employing EXAFS and XANES for similar aims, certainly it would be difficult, when not impossible, to observe the meso-shell using EXAFS spectra analysis, since the meso-shell is a less structured region than the second shell. But still there exists the possibility to study it through XANES analysis.

Figure 3 plots the K-edge XANES experimental spectrum for Pd(II) in aqueous solution, together with simulated spectra from the trajectories of Classical and Car-Parrinello MD simulations. Although both simulated spectra fit the experimental one quite well, the CPMD result reproduces better the slope of the second resonance owing to the fact that CPMD water molecules are flexible and polarizable, and thus constitute a better approximation to the behaviour of real water molecules. Given that the meso-shell is better structured in the Classical MD simulation, we can decompose the corresponding spectrum into the contributions stemming from the first hydration shell, and from the first shell plus the meso-shell atoms. Inclusion of the second solvation shell already yields the complete spectrum, so it is not plotted in the figure. There is no feature in the spectra that can be assigned to the meso-shell. However, features due to the second solvation shell can be identified. Therefore, in spite of the presence of basic structural differences between the hydration patterns of hexahydrates (spherical symmetry, detailed above) and $\mathrm{Pd}(\mathrm{II})$ tetrahydrate (crown-like plus meso-shell) their second hydration shells seem to be equivalent when approaching their study through analysis of their XANES spectra, and delicate features such as the meso-shell remain invisible to XANES scrutiny.

\section{Selecting an Intermolecular Potential on the Basis of XANES Spectra}

The structural information on anion solvation in aprotic solvents is scarce and usually the quantitative values found in the literature cover a wide range. The solvation numbers of bromide and iodide anions in aprotic and not very polar solvents are estimated in the range 1-9. It is then clear that the development of specific intermolecular potentials to be used in Computer Simulations appears as an important tool to help in structural characterization of these systems. However, due to the scarce experimental information, the validation of the developed potentials is not as easy as in the case of aqueous solutions. Figure 4 shows the experimental $\mathrm{Br} \mathrm{K}$-edge XANES spectrum of a dilute acetonitrile solution of a bromide salt recorded by Watanabe et al.[19] On the same figure the corresponding simulated XANES spectra derived from three MD simulations which employed the three $\mathrm{Br}-\mathrm{ACN}$ potentials mentioned in the Methods Section have been plotted. The comparison between simulated and experimental XANES spectra indicates that Pot. C is the most appropriate intermolecular potential. As far as the XANES agreement implicitly denotes similar structures, the analysis of the MD results give us a quantitative description of the solvation of $\mathrm{Br}^{-}$anion in acetonitrile. This halide in acetonitrile forms a rather relaxed first solvation shell formed by $\sim 10 \mathrm{ACN}$ molecules where one of the hydrogen atoms of the methyl group is placed closer to the anion, 2.7-2.9 $\AA$, than the other two hydrogen atoms of the methyl group $(c a .4 .2-4 . . \AA)$ due to the bent orientation of the ACN molecules $\left(\sim 130^{\circ}\right)$ with respect to the $\mathrm{Br}^{-}$anion.

\section{CONCLUDING REMARKS.}

The interplay between computer simulations and XAS provides a powerful tool to study ions in solution. In this paper we have shown how the comparative analysis of experimental and simulated spectra allows, for highly charged cations in solution, the determination of structural parameters of the second hydration shell accurately enough. However, features due to poor structured regions with a high dynamical component can not be identified from either experimental or simulated spectra although they appear well-defined in the Computer simulation results. Moreover, the combined use of experimental and simulated spectra can help us in the development of reliable ion-solvent interacting potentials to be used in MD simulations of ions in solution. 


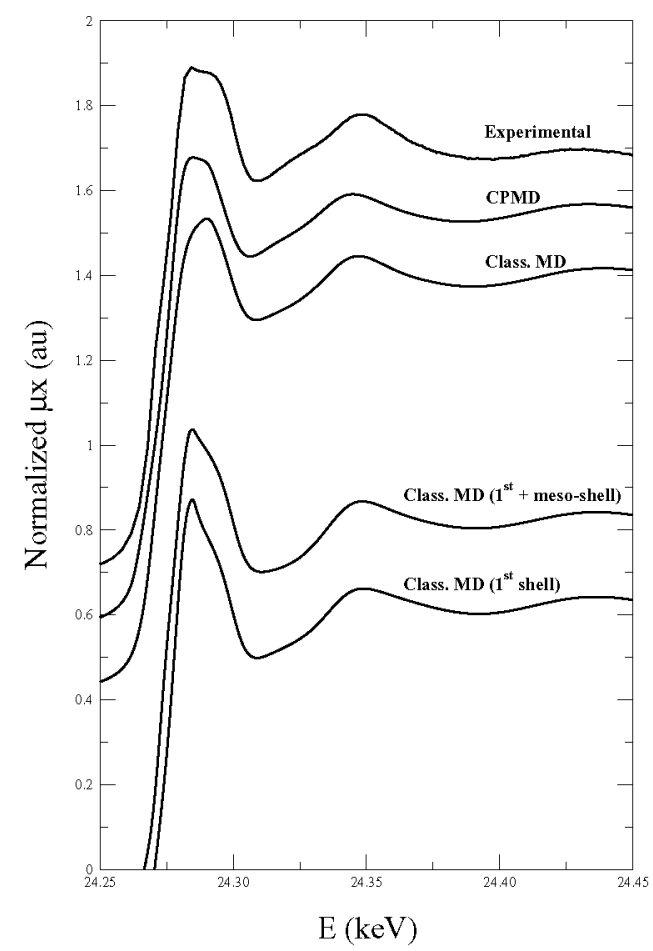

FIGURE 3. Comparison of the Pd K-edge XANES experimental and simulated spectra. The simulated ones use snapshots from a CPMD or a classical MD simulation. Changes in the features of the spectrum with the hydration shells considered in the snapshots are presented for the case of classical MD simulation.

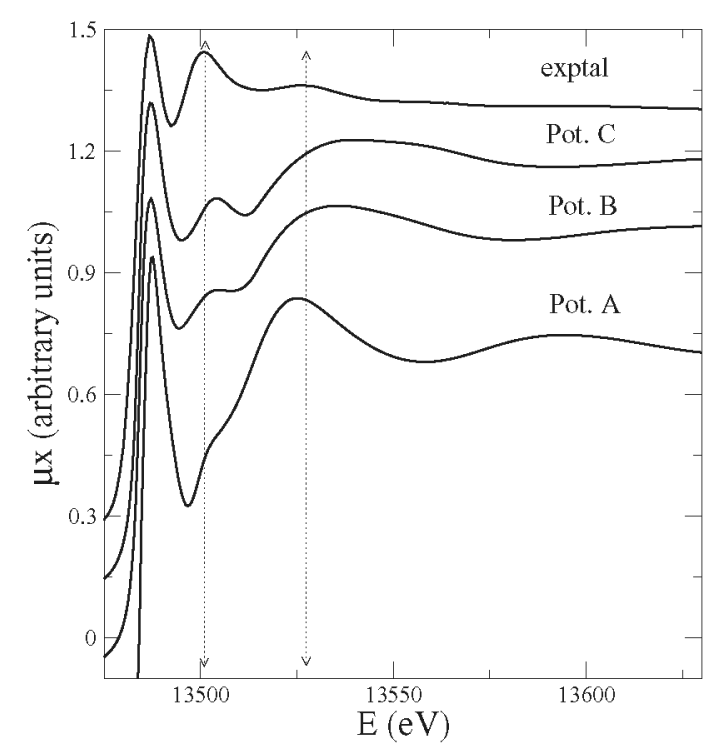

FIGURE 4. Comparison of the $\mathrm{Br} \mathrm{K}$-edge XANES experimental spectrum for bromide in acetonitrile and three simulated spectra using three different intermolecular potentials in the MD simulation.

\section{ACKNOWLEDGMENTS}

Spanish Government (CTQ2005-03657) and Junta de Andalucia (group FQM282) are acknowledged for financial support. RAE thanks CSIC for a postdoctoral I3P fellowship.

\section{REFERENCES}

1. P. J. Merkling, A. Muñoz-Páez, R. R. Pappalardo, and E. Sánchez Marcos, Phys. Rev. B 64, 092201 (2001).

2. P. J. Merkling, A. Muñoz-Páez, and E. Sánchez Marcos, J. Am. Chem. Soc. 124, 10911 (2002).

3. P. D'Angelo, O. M. Roscioni, G. Chillemi, S. Della Longa, and M. Benfatto, J. Am. Chem. Soc. 128, 1853 (2006).

4. F. Carrera, F. Torrico, D. T. Richens, A. Muñoz-Páez, J. M. Martínez, R. R. Pappalardo, and E. Sánchez Marcos, J. Phys. Chem. B 111, 8223 (2007).

5. J. M. Martínez, F. Torrico, R. R. Pappalardo, and E. Sánchez Marcos, J. Phys. Chem. B 108, 15851 (2004).

6. S. Shah, T. S. Hofer, M. Fatmi, B. Randolf, and B. Rode, Chem. Phys. Lett. 426, 301 (2006).

7. Y. Sawa, T. Miyanaga, H. Tanida, and I. Watanabe, J. Chem. Soc. Faraday Trans. 91, 4389 (1995).

8. R. R. Pappalardo, and E. Sánchez Marcos, J. Phys. Chem. 97, 4500 (1993).

9. J. M. Martínez, R. R. Pappalardo, and E. Sánchez Marcos, J. Chem. Phys. 109, 1445 (1998).

10. J. M. Martínez, R. R. Pappalardo, E. Sánchez Marcos, K. Refson, S. Díaz-Moreno, and A. Muñoz-Paez, J. Phys. Chem. B 102, 3272-3282 (1998). 
11. J. M. Martínez, P. Merkling, R. R. Pappalardo, K. Refson, and E. Sánchez Marcos, Theor. Chem. Acc. 111, 101-109 (2004).

12. E. C. Beret, R. R. Pappalardo, E. Sánchez Marcos, D. Marx, and N. L. Doltsinis, submitted (2007).

13. X. Grabuleda, C. Jaime, and P. A. Kollman, J. Comput. Chem. 21, 901 (2000).

14. T. P. Lybrand, I. Ghosh, and J. A. McCammon, J. Am. Chem. Soc. 107, 7793 (1985).

15. R. Ayala, J. M. Martínez, R. R. Pappalardo, A. Muñoz-Páez, and E. Sánchez Marcos, Mol. Simul. 32, 1035 (2006).

16. A. Ankudinov, B. Ravel, J. J. Rehr, and S. D. Conradson, Phys. Rev. B 58, 7565 (1998).

17. A. Muñoz-Páez, R. R. Pappalardo, and E. Sánchez Marcos, J. Am. Chem. Soc. 117, 11710 (1995).

18. H. Sakane, A. Muñoz-Páez, S. Díaz-Moreno, J. M. Martínez, R. R. Pappalardo, and E. Sánchez Marcos, J. Am. Chem. Soc. 120, 10397 (1998).

19. Y. Sawa, T. Miyanaga, H. Tanida, and I. Watanabe, J. Chem. Faraday Trans. 91, 4389 (1995). 